- While panoramic radiography is frequently used as a routine 'screening' examination of a new adult patient, taking posterior bitewing radiographs, in agreement with current evidence-based clinical guidelines, reduces the numbers of radiological findings and the diagnostic yield identified solely by the panoramic radiograph.

- In asymptomatic patients, radiological diagnostic yield from panoramic radiographs is lower.

- Selecting patients for panoramic radiography using clinical factors derived from the history and examination would improve the odds of achieving a high diagnostic yield.

- Alternatives to panoramic radiography (posterior bitewings and selected periapicals) can be satisfactorily used to identify abnormalities with greater diagnostic accuracy.

\title{
Screening panoramic radiography of new adult patients: diagnostic yield when combined with bitewing radiography and identification of selection criteria
}

\section{E. Rushton, ${ }^{1}$ K. Horner ${ }^{2}$ and H. V. Worthington ${ }^{3}$

\begin{abstract}
Objectives To measure the radiological diagnostic yield on screening panoramic radiographs taken of new adult patients and to identify selection criteria for panoramic radiography of new adult patients. Design Survey of 1,817 consecutive panoramic radiographs taken as 'routine' on new patients with statistical analysis of clinical and radiological findings.
\end{abstract}

Setting All radiographs were obtained from 41 general dental practitioners (GDPs). The GDPs provided the clinical information about the patient obtained by history and examination. Collection of material occurred in 1998/1999.

Materials and methods Two dental radiologists recorded the radiological findings on each of 1,817 panoramic radiographs by consensus. Those findings that would have been identified from bilateral posterior bitewing radiographs of each patient were then excluded to give modified figures for radiological findings.

Main outcome measures Indices of diagnostic yield were devised and calculated for each radiograph from the data on radiological findings. Total diagnostic yield (DY) and modified diagnostic yield (MDY), after exclusion of findings identifiable on bitewing radiographs, were both calculated. Clinical indicators of a high MDY were identified using stepwise multiple regression analysis.

Results MDY was 0 for $17 \%$ of the radiographs (all patients) and 23\% of the radiographs when the asymptomatic group were considered. The clinical variables for which the significance was high $(p<0.001)$ were: increasing number of teeth with clinical suspicion of periapical pathology, presence of partially erupted teeth, increasing number of clinically evident carious lesions, partially dentate status and presence of crowns. Conclusion Taking posterior bitewing radiographs of new adult patients would reduce the diagnostic yield identified solely by panoramic radiography. Using clinical factors derived from the history and examination as radiographic selection criteria modestly improves the odds of achieving a high diagnostic yield from panoramic radiography.

${ }^{1 *}$ Lecturer in Oral and Maxillofacial Radiology, ${ }^{2}$ Professor of Oral and Maxillofacial Radiology Imaging, ${ }^{3}$ Reader in Dental Statistics

${ }^{*}$ Correspondence to: V. E. Rushton, Radiology Department, University Dental Hospital of Manchester, Higher Cambridge Street, Manchester M15 6FH

\section{Refereed paper}

Received 14.06.01; Accepted 06.11.01

$\odot$ British Dental Journal 2002; 192: 275-279
The growth of panoramic radiography within National Health Service general dental practice in England and Wales has shown a sevenfold increase over the last two decades when compared to the growth of intra-oral radiography for the same period. ${ }^{1}$ The routine use of panoramic screening of patients continues with be used by a proportion of practitioners. ${ }^{2}$ However, justification of this procedure requires that the benefit to the patient from the examination obtained should outweigh the detriment of the exposure and that the examination should be expected to provide new information to aid the patient's management or prognosis. ${ }^{3}$

Several studies, reviewed by Rushton and Horner, ${ }^{4}$ have documented the diagnostic yield from panoramic radiography. These studies have targeted specific groups of individuals at variance with the wide spectrum of adult patients attending general dental practice. More importantly, none of these previous studies has addressed whether the panoramic yield obtained is simply duplicating that which would be evident on intra-oral radiography at a lower, or comparable, $x$-ray exposure to the patient.

Recent evidence-based guidelines ${ }^{5}$ indicate that posterior bitewing radiographs are an essential part of the assessment of a new adult dentate patient. However, there is no analogous evidence base for the use of panoramic radiography upon which to derive selection (referral) criteria suitable for British general dental practice.

The aims of the study were:

(i) to measure the diagnostic yield from screening panoramic radiographs which excluded radiological findings that would have been identified by bilateral posterior bitewing radiography and

(ii) to develop selection (referral) criteria for panoramic radiography that might identify those patients with a 'high' diagnostic yield.

\section{MATERIALS AND METHODS}

This study used a series of 1,817 screening panoramic radiographs of new adult ( $>18$ years) patients, provided by 41 general dental practitioners (GDPs) practising in England and Wales. Full details of recruitment of the GDPs and the patient sample have been reported previously. ${ }^{6}$ The study was commenced in the autumn of 1998 and concluded in the late winter of 1999. Clinical information was recorded by the GDP for each patient as outlined in Table 1. All documentation, as well as the original panoramic 


\section{Table 1 Clinical information recorded by the GDPs for each patient}

Basic patient information
Age
Sex
Year of last visit for dental treatment
Reason for attendance
Requesting complete dental care
Requesting limited treatment only
Symptoms at presentation
Asymptomatic
Pain
Swelling
Trismus
Bad taste
Other
Clinical examination findings
Number of existing restorations
Type(s) of restorations present
Simple only (amalgams, composites)
Crowns
Bridges
Partial dentures
Complete dentures
Presence of partially erupted teeth
Clinically suspected unerupted teeth
Number of clinically evident carious lesions
Presence of gingivitis
Presence of other evidence of periodontal disease
Oral Hygiene
Good
Fair
Poor
Presence of swellings
Number of teeth with clinically suspected periapical pathology
Dentition
Dentate
Partially dentate
Edentulous

radiograph, was submitted to two investigators, both dental radiologists (VER and $\mathrm{KH}$ ). Each radiograph was then viewed simultaneously by these 'experts' and a consensus radiological report prepared using the checklist shown in Table 2.

\section{Modified diagnostic yield}

The consensus radiological report of the experts formed a record of the 'radiological diagnostic yield' of each patient. However, as posterior bitewing radiography is accepted as an essential part of the radiographic assessment of a new adult dentate patient, ${ }^{5}$ it was

Table 2 Information recorded in the radiological report for each patient, based on interpretation of the panoramic radiograph

\begin{tabular}{|c|c|}
\hline Presence of calculus deposits & Yes/No \\
\hline Periodontal bone loss & $\begin{array}{l}\text { Early (up to one-third loss of bone } \\
\text { attachment) } \\
\text { Moderate (one- to two-thirds loss of bone } \\
\text { attachment) } \\
\text { Advanced (more than two-thirds loss of } \\
\text { bone attachment) }\end{array}$ \\
\hline Caries & Number of teeth with lesions present \\
\hline Periapical inflammatory pathology & Number of teeth with lesions present \\
\hline Retained roots & Number of roots* \\
\hline Unerupted teeth & Yes/No \\
\hline $\begin{array}{l}\text { Pathology affecting one/both of } \\
\text { the maxillary antra }\end{array}$ & Yes/No \\
\hline Other abnormalities & Yes/No; specific details recorded \\
\hline
\end{tabular}

${ }^{*}$ To avoid confusion, the number of units (teeth) where roots were retained was recorded, eg two retained roots of a lower molar were recorded as one retained root in the report. decided that a more realistic panoramic radiological diagnostic yield would be obtained if those findings that would have already been identified on posterior bitewing films were excluded from further consideration.

It was assumed that, for dentate patients, any pathological lesions in the posterior premolar/molar regions corresponding in area to a size $2(3 \mathrm{~cm}$ by $4 \mathrm{~cm}$ ) intra-oral film would have been identified by bitewing radiography. All caries lesions in these regions were therefore excluded from analysis, as were periodontal bone loss and presence of calculus deposits. The precise area of the dentition covered by right and left posterior bitewing radiographs was that as defined by Whaites. ${ }^{7}$ For edentulous patients, where bitewing radiography was not applicable, this modification was not performed. For partially dentate patients, the 'expert' investigators made individualised patient judgements about whether bitewing radiography would have been practicable on one, or both, sides of the mouth. Where not practicable, this modification was not performed. The resultant data was recorded on a spreadsheet for analysis using a standard statistical package. ${ }^{8}$

The radiological findings were then used to compute indices of diagnostic yield (DY) and modified diagnostic yield (MDY) for each patient, where these refer to yield before and after exclusion of findings that would have been recorded on bitewing radiographs respectively. DY and MDY were each determined in two different ways. First, DY/MDY was calculated as being the sum of the total number of lesions from each category in the checklist given in Table $2\left(\mathrm{DY}_{1} / \mathrm{MDY}_{1}\right)$. Second, MDY was defined as being the sum of the scores for positive findings in the checklist given in Table 2, where the presence of one or more lesions in any single category was given a score of 1 and the absence of a lesion in that category was given a score of $0\left(\mathrm{DY}_{2} / \mathrm{MDY}_{2}\right)$. Thus, for example, five carious lesions in an individual contributed 5 to $\mathrm{DY}_{1}$ but only 1 to $\mathrm{DY}_{2}$.

\section{Data analysis}

Mean values (with standard deviation and range) were determined for $\mathrm{DY}_{1}, \mathrm{MDY}_{1}, \mathrm{DY}_{2}$ and $\mathrm{MDY}_{2}$. These values were recalculated for the sub-group of patients who were asymptomatic at first examination. Differences between the diagnostic yield (MDY $_{1}$ or $\mathrm{MDY}_{2}$ ) for dichotomous clinical variables were compared by the independent sample $t$-test. The association between ordinal (and interval) clinical variables and these diagnostic indices were assessed by Pearson's correlation. Stepwise multiple linear regression models were fitted for these indices which included an examination of colinearity and partial plots of the residuals, histograms of standardised residuals and normal probability plots for the standardised residuals. For the stepwise methods for fitting the regression models, a probability of inclusion of 0.05 and probability of exclusion of 0.10 were used. The fit of each final model was assessed by the $\mathrm{R}^{2}$ value, which indicates how much of the variability in the diagnostic yield is explained by the independent variables. The 0.05 level of significance was used throughout.

\section{RESULTS}

\section{Radiological diagnostic yield and modified diagnostic yield}

The descriptive data for DY and MDY are shown in Table 3. MDY was 0 (zero) for $17 \%$ of the panoramic radiographs (all patients) and for $23 \%$ of the radiographs of the asymptomatic sub-group. The individual radiological findings that contributed towards DY and MDY are shown in Table 4. Posterior bitewing radiography alone would have diagnosed the full extent of caries involvement in 836 (46\%) patients. For periodontal bone loss, 1,467 (81\%) of patients' radiographs exhibited either no bone loss or bone destruction in an early stage (ie crestal bone loss) of disease progression. In both these cases, bone levels would have been evident on horizontal bitewing radiography. ${ }^{9,10}$ 
Table 3 Mean values of diagnostic yield (DY) and modified diagnostic yield (MDY) in the study (number of radiographs $=1,817$ )

\begin{tabular}{llll}
\hline & Mean & Standard deviation & Range \\
\hline DY $_{1}$ & 5.29 & 3.79 & $0-28$ \\
& 4.34 & 3.35 & $0-22$ \\
MDY $_{1}$ & 2.10 & 2.16 & $0-18$ \\
DY $_{2}$ & 1.67 & 1.87 & $0-18$ \\
& 3.06 & 1.45 & $0-8$ \\
MDY $_{2}$ & 2.69 & 1.42 & $0-7$ \\
& 1.50 & 1.06 & $0-6$ \\
& 1.28 & 0.98 & $0-5$
\end{tabular}

Figures in italics are the corresponding data for radiographs of the asymptomatic sub-group of patients $(n=732)$.

\section{Relationships between radiological diagnostic yield and clinical variables}

For each of the following dichotomous independent variables: symptoms (symptomatic versus asymptomatic), presence of crowns, presence of partial dentures, gingivitis, other evidence of periodontal disease and swellings, those with a positive clinical finding had a highly significantly $(\mathrm{p}<0.001)$ greater MDY $\left(\mathrm{MDY}_{1}\right.$ and $\mathrm{MDY}_{2}$ ) than those who did not, whilst those with partially erupted and clinically suspected unerupted teeth had a highly significantly $(\mathrm{p}<0.001)$ greater $\mathrm{MDY}_{2}$ only.

There were highly significant $(\mathrm{p}<0.001)$ correlations between all the ordinal (and interval) variables for $\mathrm{MDY}_{1}$, the strongest relationship being for number of teeth with clinically suspected periapical lesions $(R=0.45)$. Correlation coefficients were lower for $\mathrm{MDY}_{2}$, with non-significant relationships for both age and the number of restorations.

To summarise, those patients with a 'high' diagnostic yield who had not visited the dentist for a longer time, had significantly more restorations, significantly poorer oral hygiene, significantly more teeth with suspected periapical pathology and were less likely to be fully dentate.

\section{Regression analysis}

Items of missing data due to insufficient completion of the clinical proforma meant that 1,752 cases could be included in the regression analysis. Nine independent variables were included in the final stepwise regression for MDY 1 (Table 5). Eight of these variables also appear in the final model for $\mathrm{MDY}_{2}$ (Table 6). The fit of the models for both MDY $\left(\mathrm{R}^{2}=0.29\right)$ and $\mathrm{MDY}_{2}\left(\mathrm{R}^{2}=0.20\right)$ was limited, the clinical factors only explaining $29 \%$ and $20 \%$ respectively of the variability of MDY 1 and $\mathrm{MDY}_{2}$ in these patients.

The clinical variables for which the significance was high $(p<0.001)$ in both MDY $_{1}$ and $\mathrm{MDY}_{2}$ were: increasing number of teeth with clinical suspicion of periapical pathology, presence of partially erupted teeth, increasing number of clinically evident carious lesions, partially dentate status and presence of crowns. Clinical suspicion of unerupted teeth appeared as a highly significant $(\mathrm{p}<0.001)$ variable in $\mathrm{MDY}_{2}$ only.

\section{DISCUSSION}

This study had two principal aims. Firstly, to compute a diagnostic yield from screening panoramic radiographs which was modified by exclusion of positive radiological findings that would have been identified by bilateral posterior bitewing radiography. Secondly, to develop selection criteria for panoramic radiography that might identify those patients with a 'high' diagnostic yield.

As previously reported, ${ }^{6}$ this study group was an accurate and representative sample of all those adult patients undergoing panoramic radiography in general dental practice in England and Wales. Inter-practitioner bias had been eliminated by recruiting only those practitioners who had identified themselves as practising 'routine' panoramic radiography of all new adult patients. ${ }^{2}$
Table 4 Radiological findings recorded on the panoramic radiographs $(n=1,817)$ contributing to diagnostic yield (DY) and modified diagnostic yield (MDY)

\begin{tabular}{|c|c|c|}
\hline & \multicolumn{2}{|c|}{ DY (expert assessment) MDY (expert assessment) } \\
\hline & Number (\%) & Number (\%) \\
\hline Presence of calculus deposits & $961(52.9)$ & \\
\hline \multicolumn{3}{|l|}{ Periodontal bone loss } \\
\hline None & $785(43.2)$ & \\
\hline Early & $682(37.5)$ & \\
\hline Moderate & 297 (16.3) & \\
\hline Advanced & $53(3.0)$ & \\
\hline \multicolumn{3}{|l|}{ Number of caries lesions } \\
\hline None & $560(30.8)$ & $1401(77.1)$ \\
\hline 1 & $359(19.7)$ & $243(13.4)$ \\
\hline 2 & $290(16.0)$ & $96(5.3)$ \\
\hline 3 & $182(10.0)$ & $38(2.1)$ \\
\hline 4 & $127(7.0)$ & $20(1.1)$ \\
\hline 5 or more & $299(16.5)$ & 19(1.0) \\
\hline \multicolumn{3}{|l|}{ Number of periapical lesions } \\
\hline None & $1087(59.8)$ & $1087(59.8)$ \\
\hline 1 & $420(23.1)$ & $420(23.1)$ \\
\hline 2 & $162(8.9)$ & $162(8.9)$ \\
\hline 3 & $78(4.3)$ & $78(4.3)$ \\
\hline 4 or more & $70(3.9)$ & $70(3.9)$ \\
\hline \multicolumn{3}{|l|}{ Number of retained roots } \\
\hline None & $1503(82.7)$ & $1503(82.7)$ \\
\hline 1 & $230(12.7)$ & $230(12.7)$ \\
\hline 2 & $45(2.5)$ & $45(2.5)$ \\
\hline 3 or more & $39(2.1)$ & $39(2.1)$ \\
\hline Presence of unerupted teeth & $647(35.6)$ & $647(35.6)$ \\
\hline Pathology of maxillary antra & $255(14.0)$ & $255(14.0)$ \\
\hline Other abnormalities & $366(20.1)$ & $366(20.1)$ \\
\hline
\end{tabular}

Inter- and intra-examiner repeatability of expert radiological assessments have previously been reported, ${ }^{6}$ but involved re-reading of random samples of radiographs and calculation of the kappa $(\kappa)$ statistic. ${ }^{11}$ Agreement was excellent for both intra-observer and inter-observer assessments for all types of pathologies considered except for 'presence of calculus' and 'number of caries lesions' where agreement was only 'fair to good' (for intra-observer assessment) and for 'all other abnormalities' (inter-observer assessment).

The consensus of research into the efficacy of panoramic radiography in caries identification has shown that it is inferior to intraoral radiography. ${ }^{4,12,13}$ Similar findings have been reported for the panoramic assessment of periodontal bone loss. ${ }^{4,10,14,15}$ Dentists are well aware of these limitations ${ }^{2}$ but, as previously shown, ${ }^{6}$ the majority of patients (57\%) in this study had no radiographs other than the panoramic film taken. Current guidelines ${ }^{5}$ endorse the use of bitewing radiography for all new dentate patients. Bitewing radiography provides information on the degree of caries involvement as well as information on periodontal bone levels. In this study, we excluded periodontal bone loss from consideration on these grounds. Posterior bitewing radiography, either conventional

Table 5 Regression coefficients $(\beta)$ and standard errors $[S E(\beta)]$ from stepwise linear multiple regression for the dependent variable 'modified diagnostic yield' using MDY1 $(n=1,752)$

\begin{tabular}{llll}
\hline Independent variable & $\beta$ & SE $(\beta)$ & $p$-value \\
\hline Teeth with suspected periapical pathology & 1.08 & 0.08 & $<0.001$ \\
Clinically evident caries lesions & 0.77 & 0.07 & $<0.001$ \\
Presence of crowns & 0.40 & 0.10 & $<0.001$ \\
Presence of partially erupted teeth & 0.67 & 0.12 & $<0.001$ \\
Dentition & 0.18 & 0.05 & $<0.001$ \\
Oral hygiene & 0.22 & 0.07 & 0.002 \\
Swelling & 0.47 & 0.14 & 0.001 \\
Presence of bridges & 0.56 & 0.18 & 0.002 \\
Presence of partial dentures & 0.40 & 0.19 & 0.04 \\
\hline
\end{tabular}

$R^{2}=0.29$ 


\begin{tabular}{|c|c|c|c|}
\hline Independent variable & $\beta$ & $\operatorname{SE}(\beta)$ & p-value \\
\hline Teeth with suspected periapical pathology & 0.40 & 0.04 & $<0.001$ \\
\hline Presence of partially erupted teeth & 0.53 & 0.06 & $<0.001$ \\
\hline Clinically evident caries lesions & 0.28 & 0.03 & $<0.001$ \\
\hline Dentition & 0.10 & 0.03 & $<0.001$ \\
\hline Presence of crowns & 0.21 & 0.05 & $<0.001$ \\
\hline Clinically suspected unerupted teeth & 0.21 & 0.06 & $<0.001$ \\
\hline Swelling & 0.25 & 0.07 & 0.001 \\
\hline Presence of partial dentures & 0.32 & 0.10 & 0.001 \\
\hline Presence of bridges & 0.26 & 0.09 & 0.005 \\
\hline
\end{tabular}

(horizontal) or vertical can demonstrate bone levels, angular defects and furcation involvement related to the posterior teeth effectively apart from cases where remaining attachment is limited to the apical regions. Although bitewing films do not show the anterior teeth, it has been shown that premolar and molar bone levels are representative of the whole dentition with respect to marginal bone levels. ${ }^{16}$ Furthermore, when combined with film holders, the bitewing projection provides the optimal geometry to allow monitoring of disease progression, ${ }^{15,17}$ an option not available with panoramic radiography. ${ }^{18}$ In addition, the limiting resolution of panoramic radiography ${ }^{19}$ is inferior to that of intra-oral radiography. ${ }^{20}$

To perform the study we needed to identify a method of combining the various types of dental and jaw pathosis on panoramic radiographs into a unified index of diagnostic yield. This is a difficult procedure to perform because the importance of one lesion, eg a cyst, would probably be seen as far greater than another, eg a single carious lesion. The indices we developed gave each the same value in the indices. It might have been possible to apply 'quality factors' to more serious lesions to amplify their influence upon the final diagnostic yield score but, in the absence of any grounds for setting the scale of such factors, this was decided against. The second index $\left(\mathrm{DY}_{2}, \mathrm{MDY}_{2}\right)$ was a compromise that at least reduced the overwhelming effect of common dental lesions such as multiple carious teeth. However, as unequivocally serious pathology was very rare, it is probable that using a more sophisticated index of radiological yield would not have influenced the final regression analyses to a significant degree.

Whilst this study has shown the degree to which panoramic radiography duplicates the findings on bitewing radiography, a proportion of study patients did present with a high diagnostic yield. It is important to look at the value of this panoramic radiographic yield rather than presenting it as a list of common pathoses and anomalies. Adopting this method enables the development of a more realistic set of clinical indicators which will ultimately influence the management of these patients. From this study (Tables 5 and 6), it is apparent that past experience of dental disease (as shown by presence of crowns and edentulous areas) is an indicator of current disease, whilst clinical disease (symptoms, clinically evident caries and swellings) are indicators of hidden pathosis. Those with partially erupted and clinically suspected unerupted teeth had a significantly higher $(\mathrm{p}<0.001) \mathrm{MDY}_{2}$. This was due, in part, to the loss of the swamping effect of common dental pathosis negated by the method used to calculate $\mathrm{MDY}_{2}$ but also to the increased prevalence of partially erupted and clinically suspected unerupted teeth amongst younger patients who presented with reduced levels of common dental disease.

By examining Tables 5 and 6 and including only those clinical variables for which the significance value was $<0.001$, the following clinical factors were identified as being the best predictors of a useful diagnostic yield:
- Clinical suspicion of teeth with periapical pathology

- Presence of partially erupted teeth

- Clinically evident caries lesions

- Dentition

- Presence of crowns

- Clinically suspected unerupted teeth

Each of the $\mathrm{R}^{2}$ values were low, explaining only a small proportion of the variability in the indices of yield. Therefore a cautionary approach must be adopted when promoting these indicators as an absolute means of guaranteeing the clinician a high certainty of a diagnostic yield from panoramic radiography. At best, these indices would only modestly improve the odds of a positive diagnostic yield if a panoramic radiograph was taken.

Dental caries and periodontal disease account for the majority of pathology seen in patients attending for routine dental treatment, emphasising the importance of bitewing radiography for all new dentate patients. ${ }^{5}$ The findings of this study agree with other research $^{21}$ which has documented dramatic reductions in panoramic diagnostic yield when the film is combined with intraoral radiography. Importantly, bitewing radiography has been shown to be successful in alerting the practitioner to the possibility of periradicular pathology. Using the criteria of radiographic evidence of previous endodontic treatment or deep caries combined with periapical radiography of those teeth exhibiting clinical signs and symptoms, Akerblom and colleagues ${ }^{22}$ were able to detect $90 \%$ of periradicular lesions in their sample group.

Although previous studies have confined themselves to differing population groups and have used different methodologies, ${ }^{21,23,24}$ with one study ${ }^{23}$ limited to full mouth intra-oral radiography, comparisons can be made between previous work and this present study. In each of the combined studies, the number of clinically evident caries lesions have been shown to be a positive predictor of yield. With regard to periodontal yield, Douglass et al. ${ }^{24}$ found that 'periodontal pocket depth' was a significant indicator of diagnostic yield. The study by Weems and colleagues ${ }^{23}$ found to the contrary, showing limited benefit of radiography of the periodontium in general periodontal treatment planning. An expert panel, ${ }^{5}$ following an extensive review of the available literature, found insufficient research data to substantiate the development of definitive guidelines for the use of radiography in periodontal practice. The panel ${ }^{5}$ emphasised that radiography must be "viewed as secondary to a clinical examination in the diagnosis of periodontal diseases'. Finally, White et al. ${ }^{21}$ reported that the selection criterion most predictive of a high diagnostic yield was when the radiograph was taken for a 'non-screening purpose', a guideline with which this study agrees.

This study attempted to identify selection criteria for panoramic radiography based upon diagnostic yield alone. However, a superior approach would have been to address the issue of the significance of diagnostic yield to treatment outcomes. For example, a radiological finding may be identified (eg an unerupted third molar), but require no intervention by the clinician. Exclusion of such findings from diagnostic yield might have influenced the results of the regression analysis in this study. We are currently reviewing our research data in conjunction with the information provided by the participating dentists in an attempt to achieve this.

This research, along with that of others, ${ }^{25,26}$ suggests that the application of selection criteria for intra-oral radiography could effectively remove any perceived need for panoramic radiography for the majority of patients. Intensive continuing education is needed to change practitioners' current practice.

In this sample of patients from 41 general dental practices who underwent routine panoramic radiography, a number of conclusions can be drawn: 
1. Taking posterior bitewing radiographs of new adult patients, in agreement with current evidence-based clinical guidelines, reduces the number of radiological findings and the diagnostic yield identified solely by panoramic radiography.

2. When yield from posterior bitewing radiographs is removed, almost one-fifth of patients received no benefit from panoramic radiography. This rose to almost one-quarter of patients when the asymptomatic attenders were examined in isolation.

3. Using clinical factors derived from the history and examination as radiographic selection criteria modestly improves the odds of achieving a high diagnostic yield from panoramic radiography.

4.The use of panoramic radiographs in general dental practice can be questioned when valid alternatives (bitewing radiography and selected periapical examination) are readily available at lower cost and improved diagnostic accuracy.

This study was supported by a grant from the NHS North West Regional Reactive Funding Scheme. The authors would like to thank Annette Vesty for inputting the data and all the GDPs for participating in the study.

1. Tanner R J, Wall B F, Shrimpton P C, Hart D, Bungay D R. Frequency of Medical and Dental X-ray examinations in the UK-1997/98. NRPB-R320. Chilton: National Radiological Protection Board, 2001.

2. Rushton VE, Horner K, Worthington H V. Aspects of panoramic radiography in general dental practice. Br Dent J 1999; 186: 342-344.

3. National Radiological Protection Board Guidance Notes for Dental Practitioners on the Safe Use of X-ray Equipment. p 13. London: Department of Health, 2001.

4. Rushton VE, Horner K. The use of panoramic radiology in dental practice. J Dent, 1996; 24: 185-201.

5. Faculty of General Dental Practitioners (UK). Selection criteria for dental radiography. London: Faculty of General Dental Practitioners (UK), Royal College of Surgeons of England, 1998.

6. Rushton VE, Horner K, Worthington HV. Screening panoramic radiology of adults in general dental practice: radiological findings. Br Dent J, 2001; 190: 495-501.

7. Whaites E. Bitewing radiography. In: Whaites, E. Essentials of dental radiography and radiology. 2nd edn; pp 91-98. Churchill Livingstone (Medical division of Pearson Professional Ltd.): New York, 1996.

8. SPSS. SPSS for Windows Standard Version 9.0. Chicago: SPSS Inc., 1989-1999.
9. Hirschmann P N, Horner K, Rushton V E. Selection criteria for periodontal radiography. Br Dent J, 1994; 176: 324-325.

10. Tugnait $A$, Clerehugh $V$, Hirschmann, $P N$. The usefulness of radiographs in diagnosis and management of periodontal diseases: a review. J Dent, 2000; 28: 219-226.

11. Cohen J. A coefficient of agreement for nominal scales. Educ Psychol Measur, 1960; 20:37-46.

12. Molander $B, A$ Ahlqwist $M$, Grondahl H-G. Panoramic and restrictive intraoral radiography in comprehensive oral radiographic diagnosis. Eur J Oral Sci, 1995; 103 191-198.

13. Flint D J, Paunovich E, Moore W S, Wofford D T, Hermesch C B. A diagnostic comparison of panoramic and intraoral radiographs. OralSurg Oral Med Oral Pathol Oral Radiol Endod, 1998; 85: 731-735.

14. Pepelassi E A, Diamanti-Kipioti A. Selection of the most accurate method of conventional radiography for the asessment of periodontal osseous destruction. J Clin Periodontol, 1997; 24: 557-567.

15. Gutteridge $D L$. The use of radiographic techniques in the diagnosis and management of periodontal disease. Dentomaxillofacial Radiol, 1995: 24: 107-113.

16. Bjorn A L, Bjorn H, Halling A. An abbreviated index for periodontal bone height. Odontol Revy, 1975; 26: 225-231.

17. Pitts N B. The use of bitewing radiographs in the management of dental caries: scientific and practical considerations. Dentomaxillofacial Radiol, 1996; 25: 5-16.

18. Benn D K. A review of the reliability of radiographic measurements in estimating alveolar bone changes. J Clin Periodontol, 1990; 17:14-21.

19. FAXIL. Physical Evaluation of Imaging Performance of Agfa-Gevaert Screen-film Systems. Faxil Assessment Report No.22, STB 6E/85/26. London: HMSO, 1985

20. Czajka J, Rushton VE, Shearer A C and Horner K. Sensitometric and image quality performance of "rapid" intraoral film processing techniques. Br J Radiol, 1996; 69: 49-58.

21. White $S C$, Forsythe $A B$, Joseph $L$ P. Patient-selection criteria for panoramic radiography. Oral Surg Oral Med Oral Pathol, 1984; 57: 681-690.

22. Akerblom $A$, Rohlin $M$, Hasselgren $\mathrm{G}$. Individualised restricted intraoral radiography versus full-mouth radiography in the detection of periradicular lesions. Swed DentJ, 1986; 12: 151-159.

23. Weems R A, Manson-Hing L R, Jamison H C, Greer, D F. Diagnostic yield and selection criteria in complete intraoral radiography. J Am Dent Assoc, 1985; 110: 333-338.

24. Douglass C W, Valachovic R W, Berkey C S, Chauncey H H, McNeil B J. Clinical indicators of radiographically detectable dental diseases in the adult patient. Oral Surg Oral Med Oral Pathol, 1988; 65: 474-482.

25. Brooks S L, Cho S Y. Validation of a specific selection criterion for dental periapical radiography. Oral Surg Oral Med Oral Pathol, 1993; 75: 383-386.

26. Richardson PS. Selected periapical radiology compared to panoramic screening. Primary Dent Care, 1997; 4: 95-99. 